\title{
MERCADO DE TRABALHO EM SAÚDE NA REGIÃO SUDESTE-BRASIL: A INSERÇÃO DA EQUIPE DE ENFERMAGEM
}

Ana Luiza Stiebler Vieira ${ }^{1}$

Antenor Amâncio Filho ${ }^{2}$

Eliane dos Santos de Oliveira ${ }^{3}$

Vieira ALS, Amâncio A Filho, Oliveira ES. Mercado de trabalho em saúde na região sudeste-Brasil: a inserção da equipe de enfermagem. Rev Latino-am Enfermagem 2004 janeiro-fevereiro; 12(1):134-8.

O estudo tem como objetivo apontar as principais características do mercado de trabalho da enfermagem na Região Sudeste, usando como referência os dados recentes da pesquisa Assistência Médico Sanitária-AMS do Instituto Brasileiro de Geografia e Estatística-IBGE. Ocupando 272.398 empregos na região, a equipe de enfermagem trabalha majoritariamente no setor público de saúde e nos estabelecimentos hospitalares. Coerente com as políticas de descentralização de saúde implantadas no país, verifica-se, em 1999, a municipalização da força de trabalho de enfermagem e sinais de flexibilização do seu mercado. Diante deste contexto regional e nacional, recomenda-se a aplicação de uma pesquisa para analisar, quantitativa e qualitativamente, o atual mundo de trabalho da equipe de enfermagem.

DESCRITORES: enfermagem; ocupações em saúde; trabalho

THE HEALTH LABOR MARKET IN THE SOUTH-EAST OF BRAZIL: THE INSERTION OF THE NURSING TEAM

The study points out the main characteristics of the nursing labor market in the Brazilian South-East, using MedicalSanitary Assistance-AMS research data as a reference base, which were recently published by the Brazilian Institute of National Statistics and Geography-IBGE. With 272,398 jobs in this region, the nursing team mainly works in the public health system and hospital institutions. In coherence with the Brazilian health system decentralization policies, the municipalization of nursing jobs and signs of labor market flexibility were observed in 1999. In view of this national and regional context, a research should be carried to obtain a quantitative and qualitative analysis of the current labor market for the nursing team.

DESCRIPTORS: nursing; work; occupations

\section{MERCADO DE TRABAJ O EN SALUD EN LA REGIÓN SUDESTE BRASILEÑA: LA INSERCIÓN DEL EQUIPO DE ENFERMERÍA}

El estudio señala las características principales del mercado del trabajo en enfermería en la región sureste, usando como referencia los datos publicados recientemente por la Investigación de la Asistencia Médico- Sanitaria-AMS desarrollada por el Instituto Brasileño de Geografía y Estadística-IBGE. El equipo de enfermería tiene 272.398 puestos de trabajo en el sistema público de salud, sobre todo en hospitales. Consonante con la política brasileña de descentralización en salud, se observa en 1999, la municipalización de los puestos de enfermería y mayor flexibilidad de este mercado del trabajo. De acuerdo con el contexto nacional y regional, se aconseja la aplicación de una investigación para analizar, cuantitativamente y cualitativamente, el actual mercado del trabajo de enfermería.

DESCRIPTORES: enfermería; empleos en salud; trabajo

\footnotetext{
${ }^{1}$ Doutor em Enfermagem, Mestre em Saúde Coletiva, Pesquisador Associado, e-mail: stiebler@ensp.fiocruz.br; ${ }^{2}$ Doutor em Educação, Pesquisador Adjunto, e-mail: afilho@uol.com.br; ${ }^{3}$ Mestre em Saúde Pública, Pesquisador Colaborador, e-mail: oliveira@ensp.fiocruz.br. Escola Nacional de Saúde Pública da Fundação Oswaldo Cruz
} 
A saúde constitui um importante setor no mercado de trabalho do país. Como os demais serviços de consumo coletivo, encontra-se vinculada a um conjunto de determinantes políticos e econômicos que permeiam o mercado de trabalho em geral. Pode-se dizer que o dinamismo e as características dos seus empregos foram influenciados pelo crescimento do sistema produtor de serviços e pela reforma do setor, os quais provocaram mudanças significativas na estrutura ocupacional, no perfil quantitativo e qualitativo da força de trabalho em saúde.

Em 1999, do total de 1.375.238 empregos de saúde, 39,4\% (541.585) pertenciam à equipe de enfermagem ${ }^{(1)}$ que é composta por quatro categorias: os enfermeiros (de formação universitária); os técnicos de enfermagem (de escolaridade de nível médio ou correspondente a 11 anos de estudos e com habilitação profissional); os auxiliares de enfermagem (de escolaridade de nível fundamental ou correspondente a 8 anos de estudos e com qualificação profissional); e os atendentes que correspondem aos "práticos" de enfermagem*. Embora seja representativa em relação ao total de empregos em saúde no Brasil, a absorção de cada categoria de enfermagem apresentava significativas diferenças. Entre o total de postos de trabalho da equipe no país, os enfermeiros ocupavam apenas $13,0 \%$, os técnicos, $9,2 \%$, os atendentes, $15,1 \%$, os auxiliares, por sua vez, absorviam $62,7 \%$ da oferta, totalizando, esses últimos, 339.766 empregos. Somados aos médicos, os auxiliares bipolarizavam o mercado de saúde, absorvendo, juntos, $56,0 \%$ dos empregos (769.574) do setor ${ }^{(2)}$.

Como o Sudeste historicamente se destaca com o maior mercado de trabalho em saúde, particularmente da enfermagem ${ }^{(3)}$, este artigo aponta as principais características dos empregos das categorias que compõem a equipe de enfermagem na região.
A análise dar-se-á pelas informações mais recente da pesquisa Assistência Médico Sanitária- AMS desenvolvida pelo IBGE, em 1999, limitando-se aos empregos formalmente declarados pelos estabelecimentos de saúde. Assim como as demais fontes ${ }^{* *}$ do Ministério do Trabalho, - a Relação Anual de Informações SociaisRAIS e o Cadastro Geral de Empregados e Desempregados-CAGED, a AMS não detecta a ocupação profissional autônoma clássica nem o mercado informal em saúde. Apesar dessas limitações, os dados permitem categorizar o setor institucionalizado de saúde do país, destacando aspectos importantes dos empregos da enfermagem na região, contemplando a composição interna da equipe; sua distribuição por unidades federadas, esferas administrativas, e, ainda, as modalidades de vinculação da enfermagem com os estabelecimentos de saúde, constituindo-se, portanto, em uma das fontes mais importantes de análise de empregos de saúde.

\section{COMPOSIÇÃO DA EQUIPE DE ENFERMAGEM E DISTRIBUIÇÃO GEOGRÁFICA}

A Região Sudeste, a mais evoluída economicamente do país, é formada pelos estados do Espírito Santo, Minas Gerais, São Paulo e Rio de Janeiro, abrigando as três mais importantes metrópoles nacionais. Possui 42,5\% da população brasileira e é responsável por $58,7 \%$ do PIB nacional. É também a região com a maior densidade demográfica $\left(76,31\right.$ hab. $\left./ \mathrm{km}^{2}\right)$ e o mais alto índice de urbanização: $89,3 \%$ do país. A região concentrava, em 1999, mais da metade da força de trabalho em saúde, (808.000), 51,1\% dos empregos de enfermeiros; $54,4 \%$ dos empregos de técnicos; $50,4 \%$ dos empregos de auxiliares; e 46,8\% dos empregos de atendentes, detendo a equipe de enfermagem, ao todo, 272.398 postos de trabalho(1). O Sudeste, pelo

\footnotetext{
* Embora os atendentes não sejam reconhecidos desde 1986 pelo Conselho Federal de Enfermagem como uma categoria da equipe, segundo a nova legislação da educação profissional de 1997, devido à baixa escolaridade e por não possuírem formação específica, fazem parte da análise deste estudo, pois ainda representam parcela significativa da força de trabalho da enfermagem no país

** A RAIS: Relação Anual de Informações Sociais, sistema administrativo de informação do governo federal cujos dados são divulgados pelo Ministério do Trabalho, apresenta os dados principais sobre a população assalariada, formalmente com registro de trabalho, incorporada aos setores público e privado da economia brasileira

O Cadastro Geral de Empregados e Desempregados - CAGED foi criado pelo Governo Federal, através da Lei no 4.923/65, que instituiu o registro permanente de admissões e dispensa de empregados, sob o regime da Consolidação das Leis do Trabalho - CLT. Este cadastro geral serve como base para a elaboração de estudos, pesquisas, projetos e programas ligados ao mercado de trabalho, ao mesmo tempo em que subsidia a tomada de decisões para ações governamentais
} 
desempenho de sua economia, é o centro dinâmico do mercado de trabalho de saúde no país, o que corresponde, em certa medida, à própria distribuição da capacidade instalada e dos empregos de saúde, nessa região. Como conseqüência, todas as demais regiões apresentam uma subproporção de profissionais de saúde em relação à magnitude de suas populações ${ }^{(3)}$.

Como se observa na Tabela 1, na região Sudeste, a enfermagem ocupa 272.398 postos de trabalho. São
Paulo se destaca dos demais estados, com 142.043 empregos (ou $52,1 \%$ ), constituindo-se no mais importante mercado da equipe de enfermagem. O Rio de Janeiro, segundo maior mercado, oferta 67.889 (ou 25,0\%) empregos; e Minas Gerais, o terceiro mercado, oferta 54.063 (ou 19,8\%) postos de trabalho. O Espírito Santo chama atenção pelo menor quantitativo de empregos, 8.403, ofertando, assim, apenas $3,1 \%$ dos postos de trabalho para a equipe de enfermagem na região.

Tabela 1 - Empregos de enfermagem por categorias profissionais, segundo estados da região Sudeste e Brasil - 1999

\begin{tabular}{|c|c|c|c|c|c|c|c|c|c|c|}
\hline \multirow{2}{*}{$\begin{array}{c}\text { Brasil e Região } \\
\text { Sudeste }\end{array}$} & \multicolumn{2}{|c|}{ Total } & \multicolumn{2}{|c|}{ Enferm eiro } & \multicolumn{2}{|c|}{ Técnico } & \multicolumn{2}{|c|}{ Auxiliar } & \multicolumn{2}{|c|}{ A tendente } \\
\hline & V.abs & $\%$ & V.abs & $\%$ & V.abs & $\%$ & V.abs & $\%$ & V.abs & $\%$ \\
\hline B R A S IL & 541585 & 100 & 70175 & 12,9 & 45604 & 9,2 & 339766 & 62,7 & 82040 & 15,1 \\
\hline Sudeste & 272398 & 100 & 35838 & 13,1 & 26974 & 9,9 & 171194 & 62,8 & 38392 & 14,2 \\
\hline$M$ in as $G$ erais & 54063 & 100 & 5736 & 10,6 & 6473 & 12,0 & 33899 & 62,7 & 7955 & 14,7 \\
\hline Espirito Santo & 8403 & 100 & 716 & 8,5 & 2130 & 25,5 & 4177 & 49,7 & 1380 & 16,3 \\
\hline$R$ io $d e$ Janeiro & 67889 & 100 & 9517 & 14,1 & 10378 & 15,3 & 39757 & 58,6 & 8237 & 12,1 \\
\hline São $\mathrm{P}$ aulo & 142043 & 100 & 19869 & 13,9 & 7993 & 5,6 & 93361 & 65,6 & 20820 & 14,8 \\
\hline
\end{tabular}

Fonte:(1)

Ainda que haja preponderância dos empregos de auxiliares na região, a composição interna da equipe de enfermagem é bem diferenciada nos estados. Além dos auxiliares de enfermagem, que representam a maior força de trabalho em todos os estados, em São Paulo, os atendentes, seguidos dos enfermeiros, compõem praticamente quase toda a equipe. Nesse estado, os técnicos constituem apenas $5,6 \%$ da força de trabalho em enfermagem.

No Rio de Janeiro, a composição da equipe se distribui em ordem decrescente, pelos auxiliares, os técnicos, os enfermeiros, e os atendentes. Em Minas Gerais, pelos auxiliares, os atendentes, os técnicos e os enfermeiros; e no Espírito Santo, pelos auxiliares, os técnicos, os atendentes e os enfermeiros. Apesar de os atendentes não serem reconhecidos pela Lei do Exercício Profissional da Enfermagem como uma categoria profissional, por não possuírem formação específica, sua participação ainda é representativa na força de trabalho de enfermagem, ocupando, na região, $14,2 \%$ do total da oferta dos empregos da equipe.

\section{EMPREGOS PÚBLICOS, PRIVADOS E HOSPITALARES}

O setor público constitui o maior mercado de trabalho para a equipe, exceto para os técnicos de enfermagem (Tabela 2). Para os enfermeiros, já é uma característica histórica, ou seja, desde a década de $70^{(3)}$. Interessante é observar que o setor público e o setor privado absorvem, eqüitativamente, a força de trabalho dos atendentes de enfermagem (Tabela 2). Em relação à inserção da equipe de enfermagem nos estabelecimentos ambulatoriais e hospitalares, a grande característica da região constitui a concentração da oferta de empregos nos hospitais. Dessa forma, nos estados, todas as categorias de enfermagem (exceto os atendentes no Espírito Santo) têm seu mercado centrado em estabelecimento com internação, com os seguintes percentuais sobre o total de empregos em cada unidade federada: $76,8 \%$, em São Paulo, 80,0\%, no Rio de Janeiro, $66,1 \%$, em Minas Gerais e 70,1\%, no Espírito Santo.

Tabela 2 - Empregos de enfermagem por esferas administrativas, segundo categorias profissionais-Região Sudeste,1999

\begin{tabular}{|c|c|c|c|c|c|}
\hline \multirow{2}{*}{$\begin{array}{c}\text { Categorias } \\
\text { Profissiona is }\end{array}$} & \multirow{2}{*}{$\begin{array}{l}\text { Total } \\
\text { V.abs. }\end{array}$} & \multicolumn{2}{|c|}{ Privado } & \multicolumn{2}{|c|}{ P ú b lico } \\
\hline & & V.abs. & $(\%)$ & V.abs. & $(\%)$ \\
\hline Enferm eiro & 35.838 & 14.852 & 41,4 & 20.986 & 58,6 \\
\hline Té c n ico & 26.974 & 16.177 & 60,0 & 10.797 & 40,0 \\
\hline Auxiliar & 171.194 & 83.120 & 48,6 & 88.074 & 51,4 \\
\hline A tendente & 38.392 & 19.118 & 49,8 & 19.274 & 50,2 \\
\hline Total & 272.398 & 133.267 & 48,9 & 139.131 & 51,1 \\
\hline
\end{tabular}

Fonte:(1) 


\section{EMPREGOS MUNICIPAIS}

No que tange à distribuição dos empregos públicos, transparece nitidamente o processo de municipalização. Nos estados da região Sudeste, os municípios constituem, atualmente, o maior mercado para todas as categorias de enfermagem, ofertando $57,1 \%$ (79.520) do total dos postos de trabalho públicos para a enfermagem (Tabela 3). Considerando o total de empregos públicos e privados da equipe (272.398), os municípios ofertam $29,2 \%$ dos postos de trabalho.

A municipalização da força de trabalho de enfermagem, mostra-se assim, coerente com reforma do setor saúde em direção à desfederalização ou municipalização do sistema, dos serviços, e dos recursos humanos em saúde.

Tabela 3 - Empregos públicos de enfermagem por níveis administrativos, segundo categorias profissionais - Região Sudeste, 1999

\begin{tabular}{lcccccccc}
\hline \multicolumn{1}{c}{$\begin{array}{c}\text { Categorias } \\
\text { Profissionais }\end{array}$} & \multicolumn{2}{c}{ Empregos Públicos } & \multicolumn{2}{c}{ Federal } & \multicolumn{2}{c}{ Estadual } & \multicolumn{2}{c}{ Municipal } \\
\hline & V.abs & $\%$ & V.abs & $\%$ & V.abs & $\%$ & V.abs & $\%$ \\
Enfermeiro & 20.986 & 100 & 3.187 & 15,2 & 6.206 & 29,6 & 11.593 & 55,2 \\
Técnico & 10.797 & 100 & 3.089 & 28,6 & 2.282 & 21,1 & 5.426 & 50,2 \\
Auxiliar & 88.074 & 100 & 9.448 & 10,7 & 26.522 & 30,1 & 52.104 & 59,2 \\
Atendente & 19.274 & 100 & 2.620 & 13,6 & 6.257 & 32,5 & 10.397 & 53,9 \\
\hline Total & 139.131 & 100 & 18.344 & 13,2 & 41.267 & 29,7 & 79.520 & 57,1 \\
\hline
\end{tabular}

Fonte:(1)

\section{MODALIDADES DE VÍNCULO}

A pesquisa AMS-1999 inseriu uma importante variável para a análise do mercado de trabalho: as modalidades de vínculo dos profissionais da saúde com os estabelecimentos. Classificou, como vínculo próprio, aquele em que o contrato é efetuado diretamente com o estabelecimento de saúde; intermediado, em que o contrato se dá por intermédio de empresa, cooperativa ou entidades diferentes do estabelecimento; e outro, que constitui a prestação de serviços e trabalho autônomo nos estabelecimentos. Como se verifica na Tabela 4, o vínculo próprio prevalece em todas as categorias da equipe de enfermagem. Na região, 91,1\% dos empregos situam-se nesta modalidade. Entretanto, podem-se identificar sinais de flexibilização do seu mercado de trabalho, principalmente dos enfermeiros, por meio das vinculações intermediárias e outro. Assim, somados, $11,3 \%$ da força de trabalho dos enfermeiros, $8,6 \%$ dos técnicos, $9,2 \%$ dos auxiliares e 5,7\% dos atendentes, são vinculados sob formas flexibilizadas no mercado de trabalho em saúde da região.

Tabela 4 - Empregos de enfermagem por formas de vínculos que mantém com os estabelecimentos de saúde, segundo categorias profissionais - Região Sudeste, 1999

\begin{tabular}{|c|c|c|c|c|c|c|c|}
\hline \multirow{3}{*}{$\begin{array}{l}\text { Categorias } \\
\text { Profissionais }\end{array}$} & \multirow{3}{*}{$\begin{array}{c}\text { Total } \\
\text { V.abs }\end{array}$} & \multicolumn{6}{|c|}{ FORMAS DE VÍNCULOS } \\
\hline & & \multicolumn{2}{|c|}{ Próprio } & \multicolumn{2}{|c|}{ Interm ediado } & \multicolumn{2}{|c|}{ Outros } \\
\hline & & V.abs & $\%$ & V.abs & $\%$ & V.abs & $\%$ \\
\hline Enfermeiro & 35.838 & 31.783 & 88,7 & 2.374 & 6,6 & 1.681 & 4,7 \\
\hline Técnico & 26.974 & 24.639 & 91,4 & 1.360 & 5,0 & 975 & 3,6 \\
\hline Auxiliar & 171.194 & 155.412 & 90,8 & 10.455 & 6,1 & 5.327 & 3,1 \\
\hline A tendente & 38.392 & 36.213 & 94,3 & 1.440 & 3,7 & 739 & 2,0 \\
\hline Total & 272.398 & 248.047 & 91,1 & 15.629 & 5,7 & 8.722 & 3,2 \\
\hline
\end{tabular}

Fonte:(1)

Atualmente, no SUS, essas formas de vinculações flexíveis vêm sendo utilizadas pelos gestores municipais como alternativas aos padrões do regime estatutário. Mas, para a enfermagem, a instabilidade do emprego e a perda dos direitos trabalhistas podem corroborar como outro fator, entre outros já analisados ${ }^{(4)}$, para a sua rotatividade nos serviços de saúde.

\section{CONSIDERAÇÕES FINAIS}

Embora com limitações, os dados da AMS permitiram apontar as principais características do mercado de trabalho da enfermagem na região: a ainda pequena participação dos enfermeiros na equipe, os 
auxiliares, como a maior força de trabalho de enfermagem, o estado de São Paulo, como o maior mercado, a municipalização dos postos de trabalho, e, ainda, sinais de flexibilização do trabalho da enfermagem regional. Considerando as limitações das fontes disponíveis no país sobre mercado de trabalho e o contexto atual do mundo do trabalho, uma pesquisa com os profissionais possibilitaria analisar qualitativamente o tradicional mercado de trabalho em saúde, como também visualizar a inserção da enfermagem em outros e novos mercados.

\section{REFERÊNCIAS BIBLIOGRÁFICAS}

1. IBGE (BR). Estatísticas da Saúde: Assistência Médica Sanitária. Departamento de População e Indicadores Sociais. Rio de Janeiro (RJ): IBGE; 1999.

2. Stiebler ALV, Oliveira ES. A Equipe de Enfermagem no Mercado de Trabalho em Saúde no Brasil. Saúde em Debate 2001 janeiro/abril; 25(57):63-70.

3. Stiebler ALV, Oliveira ES. Mercado de Trabalho em Saúde no Brasil: Empregos para os Enfermeiros nas três Últimas Décadas. Rev Bras Enfermagem 2001 outubro/dezembro; 54(4):623-9.

4. Anselmi ML, Duarte GG, Angerami ELS. "Sobrevivência" no Emprego dos Trabalhadores de Enfermagem em uma Instituição Hospitalar Pública. Rev Latino-am Enfermagem 2001 julho; 9(4):13-8. 